\title{
Comments on 'Pulmonary hypertension could be a risk for deep vein thrombus in lower extremities after joint replacement surgery'
}

Wen Zhang ${ }^{1}$

1. Icahn School of Medicine at Mount Sinai. Manhattan, Nova York, USA

http://dx.doi.org/10.1590/1806-9282.65.7.951

Pulmonary hypertension identified the progressive elevation of pulmonary vascular resistance, which would lead to many negative effects on the venous system and relevant organs. In Rexiti et al. ${ }^{1}$ study, the authors showed that pulmonary hypertension could be a risk for deep vein thrombus of lower extremities after hip and knee replacement surgery.

In sum, 238 patients who underwent joint replacement at the First Affiliation Hospital of Xinjiang Medical University were recruited and studied. The good sample size in this study made the results therein convincing. Among the interesting results was that the incidence of deep venous thrombosis was found to be significantly lower in the normal pulmonary artery pressure group than in the pulmonary hypertension group. Also, a positive correlation was found between pulmonary artery pressure and the incidence of deep venous thrombosis. Afterward, the authors concluded that pulmonary hypertension could be a risk factor for deep venous thrombosis in the patient's lower extremities after joint replacement surgeries. From this perspective, patients with pulmonary hypertension should be informed of thrombosis prevention after discharge from hospital in order to reduce deep venous thrombosis and the mortality rate, which provides significant prognostic values in a real clinical scenario.

This study systematically investigated the association of pulmonary hypertension regarding deep venous thrombosis, which provided insights into the field. The results are based on many years of clinical work and the related clinical data generated, which have reasonably good reference merits. The work conducted is fundamental and, therefore, highly recommended to readers.

\section{REFERENCE}

1. Rexiti P, Wutiku M, Wulamu W, Bai F, Cao L. Pulmonary hypertension could be a risk for deep vein thrombus in lower extremities after joint replacement surgery. Rev Assoc Med Bras. 2019;65(7):946-950. 